\title{
Quantum Channeling Effects for 1 MeV Positrons
}

\section{Citation}

Haakenaasen, R., Lene Vestergaard Hau, J. A. Golovchenko, J. C. Palathingal, J. P. Peng, P. Asoka-Kumar, and K. G. Lynn. 1995. Quantum Channeling Effects for $1 \mathrm{MeV}$ Positrons. Physical Review Letters 75, no. 8: 1650-1653. doi:10.1103/physrevlett.75.1650.

\section{Published Version}

doi:10.1103/PhysRevLett.75.1650

\section{Permanent link}

http://nrs.harvard.edu/urn-3:HUL.InstRepos:29406266

\section{Terms of Use}

This article was downloaded from Harvard University's DASH repository, and is made available under the terms and conditions applicable to Other Posted Material, as set forth at http:// nrs.harvard.edu/urn-3:HUL.InstRepos:dash.current.terms-of-use\#LAA

\section{Share Your Story}

The Harvard community has made this article openly available.

Please share how this access benefits you. Submit a story.

\section{Accessibility}




\title{
Quantum Channeling Effects for $1 \mathrm{MeV}$ Positrons
}

\author{
R. Haakenaasen, ${ }^{1}$ Lene Vestergaard Hau, ${ }^{1,2}$ J. A. Golovchenko, ${ }^{1,2}$ J. C. Palathingal, ${ }^{3}$ J. P. Peng, ${ }^{4}$ \\ P. Asoka-Kumar, ${ }^{4}$ and K. G. Lynn ${ }^{4}$ \\ ${ }^{1}$ Department of Physics, Harvard University, Cambridge, Massachusetts 02138 \\ ${ }^{2}$ Rowland Institute for Science, Cambridge, Massachusetts 02142 \\ ${ }^{3}$ Department of Physics, University of Puerto Rico, Mayaguez, Puerto Rico 00680 \\ ${ }^{4}$ Department of Physics, Brookhaven National Laboratory, Upton, New York 11973
}

(Received 21 March 1995)

\begin{abstract}
A high resolution angular study of positrons transmitted through a thin single crystal of Si clearly reveals a detailed fine structure due to strong quantum channeling effects. The beam transmitted in the forward direction displays many features associated with dynamical diffraction effects and long coherence lengths. Calculations are presented showing that in flight annihilation of channeled positrons can serve as a solid state probe of electron and spin densities in thin crystals.
\end{abstract}

PACS numbers: 78.70.Bj, 61.80.Mk, 75.25.+Z

Channeling is the guided motion of energetic, charged particles incident on single crystals at small angles with major crystallographic planes or axes. Classical channeling has been well understood since the 1960s, when the theory applicable to heavy ions (e.g., $\alpha$ particles) was developed by Lindhard [1], and application of the channeling effect has developed into a standard tool in materials science to evaluate crystal and impurity lattice locations [2]. A comprehensive quantum mechanical description of channeling, which is required in the study of lighter particles (electrons, positrons) with nonrelativistic transverse energies, came later [3-5], and channeling radiation experiments have shown that this theory works extremely well for electrons $[4,5]$.

In contrast to channeled electrons, which are centered around the strings or planes in the crystal, the positron trajectories occupy the middle of the channels far away from the nuclei. In a classical description of positron channeling, the yield of particles transmitted in the forward direction as a function of incidence angle will depend strongly, though continuously, on the detector acceptance angle. One may expect such a description to make reasonable predictions when many Bragg peaks, associated with a quantum mechanical description of the positron propagation, fall within both the channeling critical angle and the detector acceptance angle. In our experiments only a single transmitted Bragg peak (the zeroth order) is recorded by the detector, and only a few Bragg angles are contained in the angular region associated with classical channeling. We will show that a full quantum mechanical treatment involving multiple beam dynamical diffraction effects can account for the strong diffraction peaks and dips observed in the experiment as well as for what might be called the channeling envelope.

We envision new applications of the channeling effect based upon accurate predictions of the transverse spatial and momentum distributions of channeled positrons. The focusing of channeled positrons in the interstices of a crystal can be utilized to develop a probe for studying valence electron densities and magnetic spin densities in crystals, as will be described below.

Positron channeling has been studied earlier for different ranges of energy. Andersen, Augustyniak, and Uggerhøj measured wide-angle scattering $\left(4^{\circ}-10^{\circ}\right)$ of $1 \mathrm{MeV}$ positrons in $\mathrm{Au}$, and they observed indications of fine structure due to Bragg diffraction [6]. Further scattering measurements by Pedersen, Andersen, and Augustyniak with $1.2 \mathrm{MeV}$ positrons in $\mathrm{Si}$ showed more fine structure [7], although in both of these experiments the lack of a sufficiently intense positron source resulted in a large energy spread and a relatively coarse angular resolution. Also, the scattering yields were integrated over a rather large detector angular range. Schultz et al. performed transmission experiments with $10-50 \mathrm{keV}$ positrons in $\mathrm{Si}$, and they observed channeling effects but no fine structure [8]. Neufert, Schiebel, and Clausnitzer reported on transmission experiments on $\mathrm{MgO}$ and $\mathrm{Si}$ with $5-45 \mathrm{MeV}$ positrons and electrons [9]. They saw diffraction effects in the electron measurements, but not for positrons.

Here we report on the forward transmission of $1 \mathrm{MeV}$ positrons through a thin $\mathrm{Si}$ crystal in scans across the (100), (110), and (111) planes. Forward transmission experiments have the advantage that all the positrons detected have gone through the same effective crystal thickness, while the positrons detected at larger angles may have been scattered anywhere in the crystal. The transmitted beam also has a much higher intensity than a scattered beam. Both these factors improve the ability to detect fine structure in a forward transmission experiment. Dynamical diffraction calculations were carried out, and they show very good agreement with the experimental data for all three planes. The calculations revealed a high sensitivity to crystal thickness, which shows up primarily in the central region of the angular yield curves.

The positron experiment was conducted at the Dynamitron facility at Brookhaven National Laboratory, with a $1 \mathrm{MeV}$ positron beam (FWHM $\sim 1 \mathrm{keV}$ ) [10]. The total available beam intensity was about $3 \times 10^{5} \beta^{+} / \mathrm{s}$ within 
$1^{\circ}$. Two collimators placed $3.2 \mathrm{~m}$ apart ensured an angular divergence of $0.025^{\circ}\left[0.020^{\circ}\right.$ for the (111) plane] and reduced the beam intensity to $\approx 70 \beta^{+} / \mathrm{s}$. Beam normalization was obtained periodically by magnetically deflecting the beam onto a scintillator located after the second collimator. The $\mathrm{Si}(100)$ sample was mounted on a twoaxis $(\theta, \phi)$ goniometer. The thin region of the crystal had a diameter of $2.5 \mathrm{~mm}$, while the beam size at the crystal was $1.4 \mathrm{~mm}$. The forward transmitted beam impinged on a windowless HPGe detector $2.6 \mathrm{~m}$ downstream from the $\mathrm{Si}$ crystal. The detector subtended a half angle of $0.1^{\circ}$, ensuring that only the direct, zeroth order transmitted Bragg beam would be recorded. The collection time per point in an angular scan of the crystal was typically $100 \mathrm{~s}$. A microchannel plate in combination with an intensified charge coupled device (CCD) camera was placed $0.7 \mathrm{~m}$ upstream from the $\mathrm{Ge}$ detector and allowed detection of the beams of Bragg scattered positrons.

The crystal was prepared by chemical etching of a boron doped $\mathrm{Si}(100)$ crystal $[11,12]$. This gave a fairly uniform thin region in the middle of the crystal with a thickness varying between 2450 and $3050 \AA$ as determined by Rutherford backscattering spectroscopy (RBS) with $2 \mathrm{MeV} \mathrm{He}^{+}$ions.

The experimental data for the three planes are displayed along with the calculated transmission curves in Figs. 1(a)-1(c). It is clear from the figures that each plane has its own distinct shape. The transmitted yield is plotted versus incidence angle to the given plane in units of the corresponding Bragg angle $\left[\theta_{B}(100)=0.184^{\circ}, \theta_{B}(110)=\right.$ $\left.0.130^{\circ}, \theta_{B}(111)=0.080^{\circ}\right]$. Both the overall yield envelope as well as the fine structure including Bragg diffraction are well described by the theoretical calculations to be described shortly. A number of higher order Bragg angle dips are seen along the sides of the curves. The position of these Bragg dips follows from diffraction kinematics, whereas dynamical diffraction theory is required to explain the thickness dependent magnitude of the yield.

In all three curves displayed here we see some rather prominent dips at the extremes of the scans, which are unaccounted for by the theory. These are due to nearby planes (that have not been included in the theory) since the dips could be seen to change location in similar scans displaced parallel to the plane under study. Furthermore, the crystal thickness varies over the size of the beam, so that the experimental curve is an average of the transmission curves for a range of thicknesses. Each theoretical curve displayed is an average of the calculations for six thicknesses spaced by $100 \AA$ in the region $2500-3000 \AA$, which is consistent with the thickness range measured earlier by RBS. We note that this thickness variation is $\leq$ typical pendellosung lengths [430 $\AA$ (100), $830 \AA$ (110), $1360 \AA$ (111)]. This procedure makes the calculation less sensitive to crystal thickness, although it cannot accurately reproduce the exact thickness variation over the size of the beam. In (a)

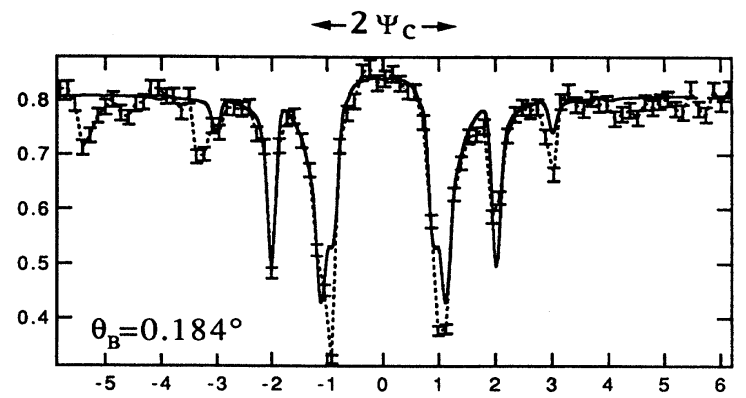

(b)

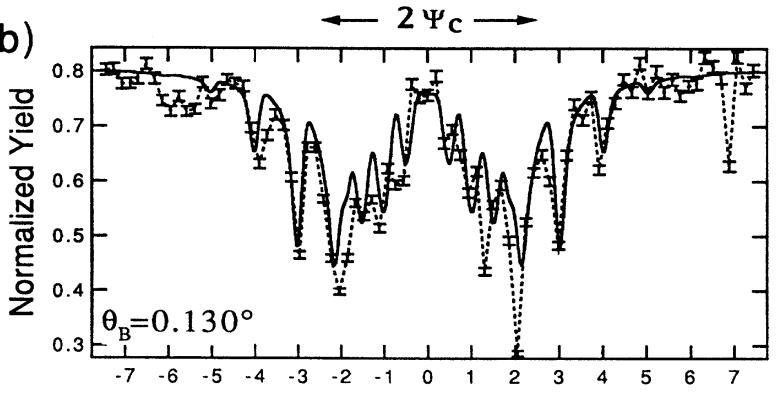

(c)

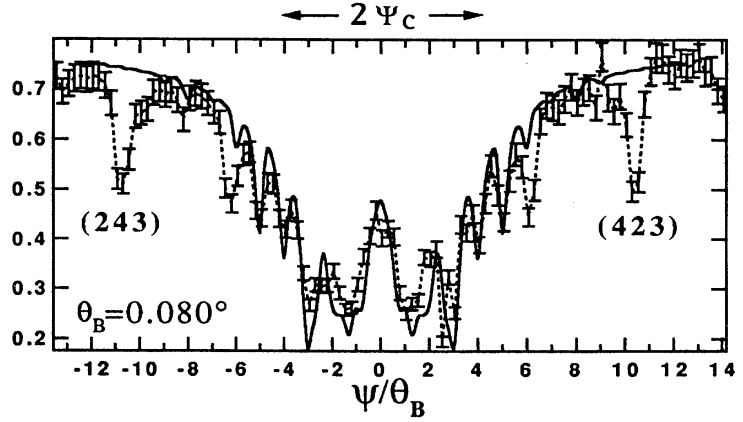

FIG. 1. Forward transmitted particle yield vs incidence angle and 21 beam calculation for the (a) (100), (b) (110), and (c) (111) planes. The distance to the nearest low index axis is (a) $6^{\circ}$ ([001]), (b) $5^{\circ}$ ([001]), and (c) $3^{\circ}([\overline{1} \overline{1} 2])$. The channeling critical angle $\Psi_{c}$ is indicated above the curves.

spite of this, the experimental and theoretical curves show very good agreement.

Complementary to the forward transmission experiment presented above is a measurement with a microchannel plate of the Bragg scattered positrons. Analysis of the angular dependence of the diffraction peaks will be published later. Here we note that the diffraction pattern from a plane is one dimensional, while the diffraction pattern from an axis is two dimensional (corresponding to momentum transfers perpendicular to the axis), as can be clearly seen in the array of diffraction spots from the $\langle 100\rangle$ axis in Fig. 2. (The forward transmitted zeroth order positrons went through the central hole in the microchannel plate and are not seen in the image.)

We use a theoretical description where the quantum motion of a positron under planar channeling conditions 


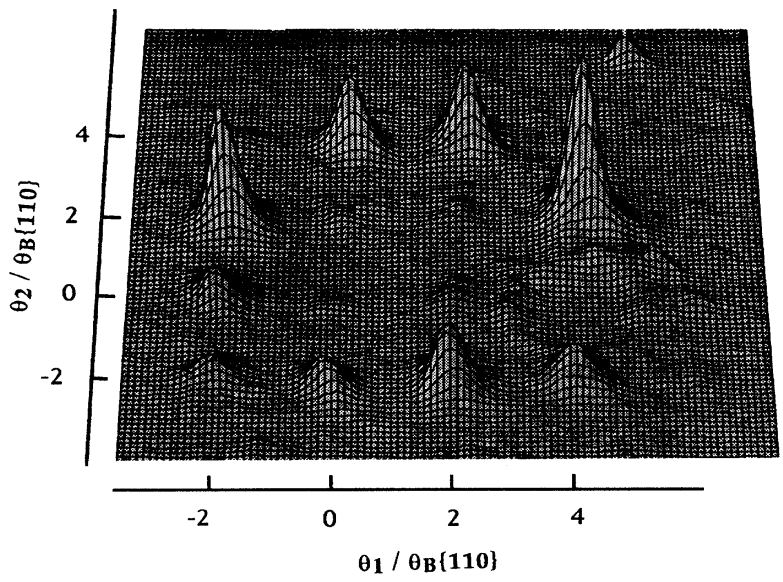

FIG. 2. Transmission diffraction pattern near the $\langle 100\rangle$ axis in $\mathrm{Si}$, displayed in units of the Bragg angle for the $\{110\}$ planes.

is governed by a one-dimensional transversely periodic continuum potential. This potential is obtained from the full crystal potential by averaging over thermal vibrations of nuclei and over the directions parallel to the plane under study. Transverse energy eigenfunctions in the crystal are chosen as simultaneous eigenfunctions of the planar transverse lattice displacement operator, i.e., Bloch states. The amplitude for transmission in the forward direction is calculated by matching a suitable linear combination of these to the incident plane wave beam on the front surface, and projecting onto the final state plane wave at the rear surface of the crystal. (We note that eigenvalues for transverse eigenstates in a continuum potential have been presented for positron propagation in Ref. [13].)

We expand the Bloch states in plane waves and typically use 21 components (each differing from the other by a transverse reciprocal lattice vector). The continuum potential is obtained as an average over a sum of atomic potentials. Thermal vibrations are taken into account through Debye-Waller factors corresponding to a vibrational amplitude of $0.0793 \AA$ [14] at room temperature. The depth dependent interference between the different eigenstates at the back of the crystal (pendellosung effect) results in fine structure which is very sensitive to crystal thickness. This interference controls the size of the dips in the transmission yield at the Bragg angles and also influences the shape of the channeling envelope [15].

A simple estimate of the effect of incoherent scattering has been included by calculating the mean free path for phonon scattering for each eigenstate $(3-10 \mu \mathrm{m}$ for the lowest states) and multiplying the corresponding phase factor by an exponential damping factor to account for depletion of the state through the crystal. The results are finally convolved with a Gaussian to take into account finite beam divergence.
An important consequence of the obtained quantitative agreement between experiment and the quantum theory is the possibility of developing a new means of probing solid state systems with positrons. Until now positron studies of solids have mainly focused on the annihilation gamma rays from positrons brought to rest in the samples. The repulsive interaction with the atomic nuclei results in a ground state positron wave function peaked in the region of the crystal occupied by valence electrons. The study of angular correlations and Doppler shifts of the annihilation gamma rays has provided valuable information about the Fermi surfaces and defects in metals $[16,17]$. However, the strong Coulombic screening of the low energy positrons by electron states in their vicinity frustrates serious attempts at quantitative measurements of electron densities in crystals. In addition, the mobility of the positrons can cause them to seek out a relatively small number of defect sites where their energy may be lowered. The characteristic decay phenomena observed may then be dominated by this effect [18].

Fast positrons, such as those used in our experiments, suffer from neither of the above effects. In addition, the positrons can be focused to probe the valence electron region via the channeling effect. The feasibility of experiments to probe the electron valence density with in flight two photon annihilation depends on the following parameters: the available positron flux with an angular divergence less than the characteristic axial channeling angle $\left(\sim 1^{\circ}\right)$, the annihilation cross section $\left[\sim\left(e^{2} / m c^{2}\right)^{2}\right]$, the electron density being probed $\left[\sim\left(h^{2} / m e^{2}\right)^{-3}\right]$, and the path length in the crystal over which a well defined positron channeling state may be maintained (dechanneling length $>1 \mu \mathrm{m}$ ). We conclude that with the available MeV-positron beam intensities of $\sim 10^{6} \mathrm{~s}^{-1}$ within a channeling critical angle, gamma counting rates of $1 \mathrm{~s}^{-1}$ can be achieved for positrons channeled in a $1.2 \mu \mathrm{m} \mathrm{Au}$ crystal. Clearly the continued development of intense positron sources will improve the prospect of a high energy in flight annihilation probe.

The ability to extract accurate electron density information requires a quantitative knowledge of the channeled positron wave function in the crystal, and our transmission experiments presented here show that this can be achieved.

A particularly exciting prospect associated with the high energy positron probe is its inherent sensitivity to electron spin polarization. Figure 3 shows a plot of the differential cross section for two photon production by a $1 \mathrm{MeV}$ (kinetic energy) longitudinally polarized positron annihilating with an electron of antiparallel (upper curve) and parallel (lower curve) spin at rest. Here $\theta$ denotes the angle between the incident positron momentum and one of the observed gamma rays. Clearly there is a very strong spin dependence for the differential cross section in the forward direction, and even for the total annihilation cross section (integrated over angle) the ratio 


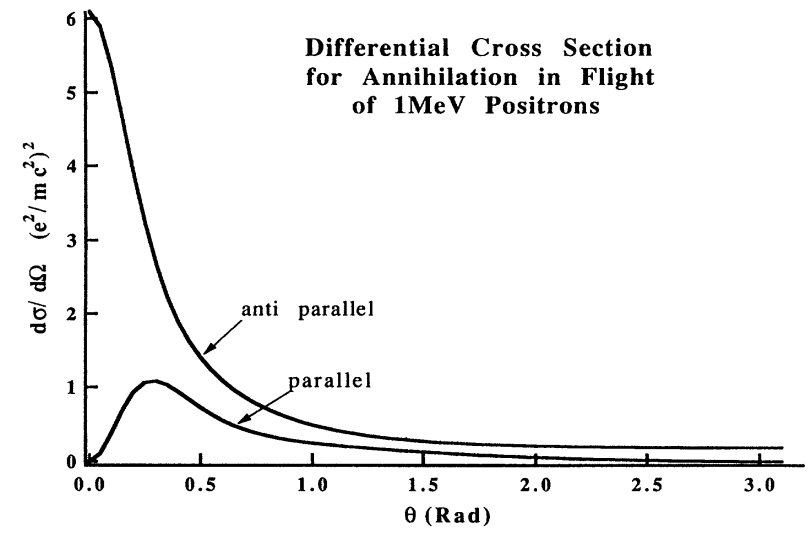

FIG. 3. Differential annihilation cross section vs angle between the incoming positron and one of the outgoing gamma rays. The two curves are for a $1 \mathrm{MeV}$ longitudinally polarized positron annihilating with an electron at rest of antiparallel (upper curve) and parallel (lower curve) spins.

of the spin antiparallel to spin parallel cross sections is 3.3 at this energy. Polarized slow positron sources have been reported by Van House and Zitzewitz [19], and their implementation in the terminal of an accelerator like the one we have used seems straightforward.

In conclusion, we have clearly observed and quantitatively accounted for dynamical diffraction effects in the forward transmission of channeled $\mathrm{MeV}$ positrons through a single crystal. The experimental scans show excellent agreement with theoretical calculations, giving us confidence that we can accurately predict the spatial and momentum distributions of channeled positrons. We further conclude that positron channeling can be developed into a new solid state probe via annihilation in flight of the channeled positrons.

We thank Harry Hacker, Walt Gibson, John Chervinsky, Don Rogers, and Mike Burns for equipment and assistance. We also thank George Kiriazides of $\mathrm{Vi}$ sion Identics Systems, Inc. for lending us a Pulnix DN007F2 intensified CCD camera with which the image in Fig. 2 was taken. The work was supported by NSF Grants No. DMR-9111494 and No. PHY-9203873, and by DOE Contract No. DE-AC02-76CH00016. R. H. acknowledges fellowship support from the Royal Norwegian Council for Scientific and Industrial Research.
[1] J. Lindhard, Mat. Fys. Medd. K. Dan. Vidensk. Selsk. 34, No. 14 (1965).

[2] L. C. Feldman, J. W. Mayer, and S. T. Picraux, Materials Analysis by Ion Channeling (Academic Press, New York, 1982).

[3] J. Lervig, J. Lindhard, and V. Nielsen, Nucl. Phys. A96, 481 (1967).

[4] J. U. Andersen, E. Bonderup, E. Lægsgaard, and A.H. Sørensen, Phys. Scr. 28, 308 (1983).

[5] Lene Vestergaard Hau and J. U. Andersen, Phys. Rev. A 47, 4007 (1993).

[6] J. U. Andersen, W. M. Augustyniak, and E. Uggerhøj, Phys. Rev. B 3, 705 (1971).

[7] M. J. Pedersen, J. U. Andersen, and W. M. Augustyniak, Radiat. Eff. 12, 47 (1972)

[8] P. J. Schultz, L. R. Logan, T. E. Jackman, and J. A. Davies, Phys. Rev. B 38, 6369 (1988).

[9] A. Neufert, U. Schiebel, and G. Clausnitzer, Radiat. Eff. 26, 49 (1975).

[10] P. Asoka-Kumar, J. S. Greenberg, S. D. Henderson, H. Huomo, M. S. Lubell, K. G. Lynn, R. Mayer, S. McCorkle, J. McDonough, J.C. Palathingal, B.F. Philips, A. Vehanen, M. Weber, and X.Y. Wu, Nucl. Instrum. Methods Phys. Res., Sect. A 337, 3 (1993).

[11] K. E. Petersen, Proc. IEEE 70, 420 (1982).

[12] K.E. Bean, IEEE Trans. Electron Devices ED-25, 1185 (1978).

[13] H. D. Dulman et al. Phys. Rev. B 48, 3818 (1993).

[14] L. V. Hau, E. Laegsgaard, and J. U. Andersen, Nucl. Instrum. Methods Phys. Res., Sect. B 48, 244 (1990); L. V. Hau, Ph.D. thesis, Aarhus University, 1990.

[15] A classical analysis of the positron transmission yield (using the concept of statistical equilibrium introduced by Lindhard [1]) predicts yield curves that look qualitatively like the envelope curves in Fig. 1; they have a central peak surrounded by a broad dip extending out to the vicinity of the classical channeling angle. However, this qualitative agreement is obtained only with a "quantum mechanical" detector solid angle corresponding to $2 \theta_{B}$. Details of this analysis will be presented elsewhere.

[16] Positrons in Solids, edited by P. Hautojärvi (SpringerVerlag, New York, 1979).

[17] Positron Solid-State Physics, edited by W. Brandt and A. Dupasquier (North-Holland, Amsterdam, 1983).

[18] P. J. Schultz and K. G. Lynn, Rev. Mod. Phys. 60, 701 (1988).

[19] J. Van House and P. W. Zitzewitz, Phys. Rev. A 29, 96 (1984). 